\title{
Generalized Strong Intuitionstic Fuzzy Hypergraph
}

\author{
T.K. Samanta* And Sumit Mohinta
}

\begin{abstract}
In this paper, we have generalized the concept of intuitionistic fuzzy hypergraph, strong intuitionistic fuzzy hypergraph by considering intuitionistic fuzzy vertex instead of crisp vertex set and interrelations between intuitionistic fuzzy vertices and family of intuitionistic fuzzy edges. A few properties of such graphs are established here.
\end{abstract}

\section{INTRODUCTION}

In 1736, Euler first introduced the concept of graph theory. The theory of graph is an extremely useful tool for solving combinatorial problems in different areas such as geometry, algebra, number theory, topology, operation research, optimization and computer science.

In 1965, Zadeh [6] published his seminal paper on "Fuzzy sets" which described fuzzy set theory and consequently fuzzy logic. The fuzzy sets give the degree of membership of an element in a given set.

In 1986, Atanassov [4] introduced the concept of intuitionistic fuzzy sets as a generalization of fuzzy sets. In the definition of intuitionistic fuzzy set, Atanassov added a new component, which determines the degree of nonmembership of an element in a given set. Intuitionistic fuzzy sets have been applied in a wide variety of fields including computer science, engineering, mathematics, medicine, chemistry, economics etc. [5, 9].

In 1975, Rosenfeld [2] introduced the concept of fuzzy graphs. The fuzzy relations between fuzzy sets were also considered by Rosenfeld and developed the structure of fuzzy graphs, obtaining analogs of several graph theoretical concepts. Atanassov [5] introduced the concept of intuitionistic fuzzy relations and intuitionistic fuzzy graphs. Strong intuitionistic fuzzy graphs is introduced in [7].

The hypergraphs was introduced by Berge [3] and has been considering as a useful tool to analyze the structure of a system. The notion of hypergraphs

2010 Mathematics Subject Classification. Primary 05C65, 05C72, Secondary 03E72.

Key words and phrases. Generalized intuitionistic fuzzy hypergraph, strong intuitionistic fuzzy hypergraph, cartesian product of hyper graph, union of hyper graph.

${ }^{*}$ Corresponding Author. 
has been extended in the fuzzy theory and concept of fuzzy hypergraphs was provided by Kaufmann [1].

Lots of works on fuzzy hypergraph $[8,9]$ have been carried out and all of them have considered the vertex set as a crisp set. In fact, in the definition of fuzzy graph, both the concepts of vertex and edges are fuzzy and there is an interrelation between the fuzzy vertex and fuzzy edges. In this paper, we have generalized the concept of intuitionistic fuzzy hypergraph by considering intuitionistic fuzzy vertex instead of crisp vertex set and an interrelation between intuitionistic fuzzy vertex and family of intuitionistic fuzzy edges. Generalized intuitionistic fuzzy hypergraph, strong intuitionistic fuzzy hypergraph and a few operations on them are defined here. Also some of their properties are studied.

\section{Preliminaries}

Definition 2.1. An intuitionistic fuzzy set (in short form IFS) $A$ in $X$ is defined as an object of the form $A=\left\{\left(x, \mu_{A}(x), \nu_{A}(x)\right): x \in X\right\}$ where the functions $\mu_{A}: X \rightarrow[0,1]$ and $\nu_{A}: X \rightarrow[0,1]$ define the degree of membership and the degree of non- membership of the element $x \in X$ respectively.

Definition 2.2. The support of an $\operatorname{IFS} A$, denoted by $\operatorname{Supp}(A)$, is defined as $\operatorname{Supp}(A)=\left\{x: \mu_{A}(x)>0, \nu_{A}(x)>0\right\}$.

Definition 2.3. [10] The intuitionistic fuzzy hypergraphs $(I F G H)$ is an ordered pair $H=(V, E)$, where

(i) $V=\left\{x_{1}, x_{2}, \ldots, x_{n}\right\}$ is a finite set of vertices;

(ii) $E=\left\{E_{1}, E_{2}, \ldots, E_{m}\right\}$ is a family of intuitionistic fuzzy subsets of $V$

(iii) $E_{j}=\left\{\left(x_{i}, \mu_{j}\left(x_{i}\right), \nu_{j}\left(x_{i}\right)\right): \mu_{j}\left(x_{i}\right)+\nu_{j}\left(x_{i}\right) \leq 1\right\}$ for all $i=1,2, \ldots, n$ and $j=1,2, \ldots, m$;

(iv) $E_{j} \neq \phi, \quad j=1,2, \ldots, m$;

(v) $\bigcup_{j} \operatorname{Supp}\left(E_{j}\right)=V, j=1,2, \ldots, m$.

Here, the edges $E_{j}$ are IFS of vertices, $\mu_{j}\left(x_{i}\right)$ and $\nu_{j}\left(x_{i}\right)$ denote the degree of membership and non-membership of vertex $x_{i}$ corresponding to the edge $E_{j}$.

\section{Generalized Strong intuitionistic FUZZy hyPERGRAPH}

We now introduce the definition of Generalized intuitionistic fuzzy hypergraphs.

Definition 3.1. The Generalized intuitionistic fuzzy hypergraph $(G I F H G)$ is an ordered pair $H=(V, E)$ where

(i) $V=\left\{x_{1}, x_{2}, \ldots, x_{n}\right\}$ is a finite set of vertices;

(ii) $\rho, \sigma: V \rightarrow[0,1]$ (intuitionistic fuzzy vertices); 
(iii) $E=\left\{E_{1}, E_{2}, \ldots, E_{m}\right\}$ is a family of intuitionistic fuzzy subsets of $V$

(iv) $E_{j}=\left\{\left(x_{i}, \mu_{j}\left(x_{i}\right), \nu_{j}\left(x_{i}\right)\right): \mu_{j}, \nu_{j}: V \rightarrow[0,1]\right\}$ with

$$
\bigvee_{j=1}^{m} \mu_{j}\left(x_{i}\right) \leq \rho\left(x_{i}\right), \quad \bigwedge_{j=1}^{m} \nu_{j}\left(x_{i}\right) \geq \rho\left(x_{i}\right)
$$

for all $i=1,2, \ldots, n$ and $j=1,2, \ldots, m$;

(v) $E_{j} \neq \phi, j=1,2, \ldots, m$;

(vi) $\bigcup_{j} \operatorname{Supp}\left(E_{j}\right)=V, j=1,2, \ldots, m$.

Here $E=\left\{E_{1}, E_{2}, \ldots, E_{m}\right\}$ can be expressed by the following matrix given by the Table 1 .

TABLE 1. Incidence Matrix $M_{H}$.

\begin{tabular}{|c||cccc|}
\hline & $E_{1}$ & $E_{2}$ & $\cdots$ & $E_{m}$ \\
\hline \hline$x_{1}$ & $\left(\mu_{1}\left(x_{1}\right), \nu_{1}\left(x_{1}\right)\right)$ & $\left(\mu_{2}\left(x_{1}\right), \nu_{2}\left(x_{1}\right)\right)$ & $\cdots$ & $\left(\mu_{m}\left(x_{1}\right), \nu_{m}\left(x_{1}\right)\right)$ \\
$x_{2}$ & $\left(\mu_{1}\left(x_{2}\right), \nu_{1}\left(x_{2}\right)\right)$ & $\left(\mu_{2}\left(x_{2}\right), \nu_{2}\left(x_{2}\right)\right)$ & $\cdots$ & $\cdots$ \\
$\vdots$ & $\vdots$ & $\vdots$ & $\vdots$ & $\vdots$ \\
$x_{n}$ & $\ldots$ & $\ldots$ & $\cdots$ & $\left(\mu_{m}\left(x_{n}\right), \nu_{m}\left(x_{n}\right)\right)$ \\
\hline
\end{tabular}

The matrix given by the Table 1 is called incidence matrix corresponding to the GIFHG $H$ and it will be denoted by $M_{H}$.

Example 3.2. Consider $H=(V, E)$ where $V=\left\{x_{1}, x_{2}, x_{3}, x_{4}\right\}$ and $E=$ $\left\{E_{1}, E_{2}, \ldots, E_{6}\right\}$. Here $\rho, \sigma: V \rightarrow[0,1]$ defined by $\rho\left(x_{1}\right)=.7, \rho\left(x_{2}\right)=.82$, $\rho\left(x_{3}\right)=.65, \rho\left(x_{4}\right)=.8$ and $\sigma\left(x_{1}\right)=.1, \sigma\left(x_{2}\right)=.09, \sigma\left(x_{3}\right)=0, \sigma\left(x_{4}\right)=.1$.

The corresponding incidence matrix $M_{H}$ is given by Table 2 .

TABLE 2. Incidence Matrix corresponding to example (3.2).

\begin{tabular}{|c||cccccc|}
\hline$M_{H}$ & $E_{1}$ & $E_{2}$ & $E_{3}$ & $E_{4}$ & $E_{5}$ & $E_{6}$ \\
\hline \hline$x_{1}$ & $(.2, .4)$ & $(0, .2)$ & $(.6, .4)$ & $(.3, .5)$ & $(.1, .3)$ & $(.1, .2)$ \\
$x_{2}$ & $(.1, .6)$ & $(.5, .4)$ & $(.7, .1)$ & $(.6, .2)$ & $(.8, .1)$ & $(.4, .1)$ \\
$x_{3}$ & $(.2, .8)$ & $(.6, .3)$ & $(.3, .4)$ & $(.1, .1)$ & $(.6, .0)$ & $(.5, .1)$ \\
$x_{4}$ & $(0, .3)$ & $(.3, .2)$ & $(.2, .6)$ & $(.4, .2)$ & $(.3, .5)$ & $(.7, .3)$ \\
\hline
\end{tabular}

Here it is easy to see that $H=(V, E)$ is a $G I F H G$.

Definition 3.3. A generalized intuitionistic fuzzy hypergraph $H=(V, E)$ is called generalized strong intuitionistic fuzzy hypergraph $(G S I F H G)$ 
if

$$
\bigvee_{j=1}^{m} \mu_{j}\left(x_{i}\right)=\rho\left(x_{i}\right) \text { and } \bigwedge_{j=1}^{m} \nu_{j}\left(x_{i}\right)=\rho\left(x_{i}\right)
$$

for all $i=1,2, \ldots, n$.

Example 3.4. Consider $H=(V, E)$ where $V=\left\{x_{1}, x_{2}, x_{3}, x_{4}\right\}$ and $E=$ $\left\{E_{1}, E_{2}, \ldots, E_{5}\right\}$. Here $\rho, \sigma: V \rightarrow[0,1]$ given by $\rho\left(x_{1}\right)=.5, \rho\left(x_{2}\right)=.6$, $\rho\left(x_{3}\right)=0$ and $\sigma\left(x_{1}\right)=.3, \sigma\left(x_{2}\right)=.2, \sigma\left(x_{3}\right)=.46$.

The corresponding incidence matrix $M_{H}$ is given by Table 3 .

TABLE 3. Incidence Matrix corresponding to example (3.4).

\begin{tabular}{|c||ccccc|}
\hline$M_{H}$ & $E_{1}$ & $E_{2}$ & $E_{3}$ & $E_{4}$ & $E_{5}$ \\
\hline \hline$x_{1}$ & $(.3, .4)$ & $(.5, .51)$ & $(.31, .3)$ & $(.4, .7)$ & $(.39, .63)$ \\
$x_{2}$ & $(0, .51)$ & $(.15, .6)$ & $(.22, .5)$ & $(.4, .54)$ & $(.6, .2)$ \\
$x_{3}$ & $(0, .63)$ & $(0, .5)$ & $(0, .47)$ & $(0, .46)$ & $(0, .49)$ \\
\hline
\end{tabular}

It is easy to verify that $H$ is strong generalized intuitionistic fuzzy hypergraph.

Definition 3.5. Let $G=(V, E)$ be a generalized intuitionistic fuzzy hypergraph, where $\rho, \sigma: V \rightarrow[0,1]$ and $E=\left\{\left(\mu_{j}, \nu_{j}\right): V \rightarrow[0,1]: j=\right.$ $1,2, \ldots, m\}$. Again, let $H=\left(V, E^{\prime}\right)$ where, $\rho^{\prime}, \sigma^{\prime}: V \rightarrow[0,1]$ and $E^{\prime}=$ $\left\{\left(\mu_{j}^{\prime}, \nu_{j}^{\prime}\right): V \rightarrow[0,1]: j=1,2, \ldots, m\right\}$.

Now $H$ is called sub graph of $G$ if

$$
\bigvee_{j=1}^{m} \mu_{j}^{\prime}\left(x_{i}\right) \leq \bigvee_{j=1}^{m} \mu_{j}\left(x_{i}\right), \quad \rho^{\prime}\left(x_{i}\right) \leq \rho\left(x_{i}\right)
$$

and

$$
\bigwedge_{j=1}^{m} \nu_{j}^{\prime}\left(x_{i}\right) \geq \bigwedge_{j=1}^{m} \nu_{j}\left(x_{i}\right), \quad \sigma^{\prime}\left(x_{i}\right) \geq \sigma\left(x_{i}\right) .
$$

A $G I F H G H=\left(V, E^{\prime}\right)$ is said to be a spanning intuitionistic fuzzy sub hypergraph of $G=(V, E)$ if $\rho^{\prime}\left(x_{i}\right)=\rho\left(x_{i}\right)$ and $\sigma^{\prime}\left(x_{i}\right)=\sigma\left(x_{i}\right)$.

Example 3.6. Consider GIFHGs $G=(V, E)$ and $H=\left(V, E^{\prime}\right), S=\left(V, E^{\prime \prime}\right)$ such that $V=\left\{x_{1}, x_{2}\right\}$. Here, $\rho, \sigma: V \rightarrow[0,1], \rho^{\prime}, \sigma^{\prime}: V \rightarrow[0,1], \rho^{\prime \prime}, \sigma^{\prime \prime}:$ $V \rightarrow[0,1]$ are defined by $\rho\left(x_{1}\right)=.5, \rho\left(x_{2}\right)=.1$ and $\sigma\left(x_{1}\right)=.3, \sigma\left(x_{2}\right)=.4$; $\rho^{\prime}\left(x_{1}\right)=.4, \rho^{\prime}\left(x_{2}\right)=.05$ and $\sigma^{\prime}\left(x_{1}\right)=.35, \sigma^{\prime}\left(x_{2}\right)=.5 ; \rho^{\prime \prime}\left(x_{1}\right)=.5$, $\rho^{\prime \prime}\left(x_{2}\right)=.1$ and $\sigma^{\prime \prime}\left(x_{1}\right)=.3, \sigma^{\prime \prime}\left(x_{2}\right)=.4$.

The corresponding incidence matrices $M_{G}, M_{H}$ and $M_{S}$ are described by 


\begin{tabular}{|c||cc|}
\hline$M_{G}$ & $E_{1}$ & $E_{2}$ \\
\hline \hline$x_{1}$ & $(.1, .3)$ & $(.4, .2)$ \\
$x_{2}$ & $(.06, .7)$ & $(.0, .25)$ \\
\hline
\end{tabular}$\quad$\begin{tabular}{|c|cc|}
\hline$M_{H}$ & $E_{1}^{\prime}$ & $E_{2}^{\prime}$ \\
\hline$x_{1}$ & $(.09, .4)$ & $(.3, .3)$ \\
$x_{2}$ & $(.03, .71)$ & $(.0, .4)$ \\
\hline
\end{tabular}

\begin{tabular}{|c||cc|}
\hline$M_{S}$ & $E_{1}^{\prime \prime}$ & $E_{2}^{\prime \prime}$ \\
\hline \hline$x_{1}$ & $(.2, .11)$ & $(.35, .15)$ \\
$x_{2}$ & $(.03, .6)$ & $(.05, .48)$ \\
\hline
\end{tabular}

Here both $H$ and $S$ are generalize intuitionistic fuzzy sub hypergraph but $S$ is spanning intuitionistic fuzzy sub hypergraph.

Definition 3.7. Let $G=(V, E)$ be a generalized strong intuitionistic fuzzy hypergraph $(G S I F H G)$, where $\rho, \sigma: V \rightarrow[0,1]$ and $E=\left\{\left(\mu_{j}, \nu_{j}\right): V \rightarrow\right.$ $[0,1]: j=1,2, \ldots, m\}$. Again, let $H=\left(V, E^{\prime}\right)$ where, $\rho^{\prime}, \sigma^{\prime}: V \rightarrow[0,1]$ and $E^{\prime}=\left\{\left(\mu_{j}^{\prime}, \nu_{j}^{\prime}\right): V \rightarrow[0,1]: j=1,2, \ldots, m\right\}$.

Now $H$ is called generalized strong intuitionistic fuzzy sub hypergraph of $G$ if

$$
\bigvee_{j=1}^{m} \mu_{j}^{\prime}\left(x_{i}\right)=\bigvee_{j=1}^{m} \mu_{j}\left(x_{i}\right), \quad \rho^{\prime}\left(x_{i}\right)=\rho\left(x_{i}\right)
$$

and

$$
\bigwedge_{j=1}^{m} \nu_{j}^{\prime}\left(x_{i}\right)=\bigwedge_{j=1}^{m} \nu_{j}\left(x_{i}\right), \quad \sigma^{\prime}\left(x_{i}\right)=\sigma\left(x_{i}\right) .
$$

A generalized strong intuitionistic fuzzy sub hypergraph $H=\left(V, E^{\prime}\right)$ is said to be a generalized strong spanning intuitionistic fuzzy sub hypergraph of $G=(V, E)$ if $\rho^{\prime}\left(x_{i}\right)=\rho\left(x_{i}\right)$ and $\sigma^{\prime}\left(x_{i}\right)=\sigma\left(x_{i}\right)$.

Example 3.8. Consider GIFHGs $G=(V, E)$ and $H=\left(V, E^{\prime}\right), S=\left(V, E^{\prime \prime}\right)$ such that $V=\left\{x_{1}, x_{2}\right\}$. Here, $\rho, \sigma: V \rightarrow[0,1], \rho^{\prime}, \sigma^{\prime}: V \rightarrow[0,1]$, $\rho^{\prime \prime}, \sigma^{\prime \prime}: V \rightarrow[0,1]$ are defined by $\rho\left(x_{1}\right)=.5, \rho\left(x_{2}\right)=.29$ and $\sigma\left(x_{1}\right)=.35$, $\sigma\left(x_{2}\right)=.42 ; \rho^{\prime}\left(x_{1}\right)=.5, \rho^{\prime}\left(x_{2}\right)=.29$ and $\sigma^{\prime}\left(x_{1}\right)=.35, \sigma^{\prime}\left(x_{2}\right)=.42$; $\rho^{\prime \prime}\left(x_{1}\right)=.5, \rho^{\prime \prime}\left(x_{2}\right)=.29$ and $\sigma^{\prime \prime}\left(x_{1}\right)=.35, \sigma^{\prime \prime}\left(x_{2}\right)=.42$.

The corresponding incidence matrix $M_{G}, M_{H}$ and $M_{S}$ are follows:

\begin{tabular}{|c||cc||c||c|}
\hline$M_{G}$ & $E_{1}$ & $E_{2}$ \\
\hline \hline$x_{1}$ & $(.31, .8)$ & $(.5, .35)$ \\
$x_{2}$ & $(.06, .7)$ & $(.29, .42)$ \\
\hline \hline$x_{H}$ & $E_{1}^{\prime}$ & $E_{2}^{\prime}$ \\
\hline$x_{2}$ & $(.5, .4)$ & $(.3, .35)$ \\
& & & \\
\end{tabular}

\begin{tabular}{|c||cc|}
\hline$M_{S}$ & $E_{1}^{\prime \prime}$ & $E_{2}^{\prime \prime}$ \\
\hline \hline$x_{1}$ & $(.5, .35)$ & $(.35, .5)$ \\
$x_{2}$ & $(.03, .42)$ & $(.29, .48)$ \\
\hline
\end{tabular}


It is easy to verify that $H$ is a generalized strong intuitionistic fuzzy sub hypergraph of $G$ and $S$ is a generalized strong spanning intuitionistic fuzzy sub hypergraph of $G$.

Definition 3.9. Consider two generalized strong intuitionistic fuzzy hypergraphs $H_{1}=\left(V_{1}, E_{1}\right)$ and $H_{2}=\left(V_{2}, E_{2}\right)$ where $V_{1}=\left\{x_{1}, x_{2}, \ldots, x_{n}\right\}$, $V_{2}=\left\{y_{1}, y_{2}, \ldots, y_{m}\right\}, \rho_{1}, \sigma_{1}: V_{1} \rightarrow[0,1], \rho_{2}, \sigma_{2}: V_{2} \rightarrow[0,1]$ and $E_{1}=$ $\left\{\left(\mu_{11}, \nu_{11}\right),\left(\mu_{12}, \nu_{12}\right), \ldots,\left(\mu_{1 k}, \nu_{1 k}\right)\right\}, E_{2}=\left\{\left(\mu_{21}, \nu_{21}\right)\right.$, $\left.\left(\mu_{22}, \nu_{22}\right), \ldots,\left(\mu_{2 p}, \nu_{2 p}\right)\right\}, \mu_{1 i}, \nu_{1 i}: V_{1} \rightarrow[0,1] ; \mu_{2 j}, \nu_{2 j}: V_{2} \rightarrow[0,1]$ for all $i=1,2, \ldots, k$ and $j=1,2, \ldots, p$.

The Cartesian product $H_{1} \times H_{2}$ of $H_{1}$ and $H_{2}$ is defined as a pair $\left(V_{1} \times\right.$ $\left.V_{2}, E_{1} \times E_{2}\right)$ where
(i) $\left\{\begin{array}{l}\left(\rho_{1} \times \rho_{2}\right)(x, y)=\rho_{1}(x) \wedge \rho_{2}(y) \\ \left(\sigma_{1} \times \sigma_{2}\right)(x, y)=\sigma_{1}(x) \vee \sigma_{2}(y)\end{array} \quad\right.$ for all $x \in V_{1}$ and $y \in V_{2} ;$
(ii) $\begin{cases}\left(\mu_{1 i} \times \mu_{2 j}\right)(x, y)=\mu_{1 i}(x) \wedge \mu_{2 j}(y) & \text { for all } x \in V_{1}, y \in V_{2}, \\ \left(\nu_{1 i} \times \nu_{2 j}\right)(x, y)=\nu_{1 i}(x) \vee \nu_{2 j}(y) & j=1,2, \ldots, k \text { and }\end{cases}$

Remark 3.10. If both $H_{1}$ and $H_{2}$ are not strong then $H_{1} \times H_{2}$ may or may not be strong, which is verified by the following example.

Example 3.11. Consider $H_{1}=\left(V_{1}, E_{1}\right)$ and $H_{2}=\left(V_{2}, E_{2}\right)$ where $V_{1}=$ $\left\{v_{1}^{\prime}, v_{2}^{\prime}\right\}, E_{1}=\left\{E_{1}^{\prime}, E_{2}^{\prime}\right\}$ and $V_{2}=\left\{v_{1}^{\prime \prime}, v_{2}^{\prime \prime}\right\}, E_{2}=\left\{E_{1}^{\prime \prime}, E_{2}^{\prime \prime}\right\}$. Here $\rho_{1}, \sigma_{1}$ : $V_{1} \rightarrow[0,1], \rho_{2}, \sigma_{2}: V_{2} \rightarrow[0,1]$ are defined by $\rho_{1}\left(v_{1}^{\prime}\right)=.3, \rho_{1}\left(v_{2}^{\prime}\right)=.5$, $\sigma_{1}\left(v_{1}^{\prime}\right)=.2, \sigma_{1}\left(v_{2}^{\prime}\right)=.4$ and $\rho_{2}\left(v_{1}^{\prime \prime}\right)=.45, \rho_{2}\left(v_{2}^{\prime \prime}\right)=.95, \sigma_{2}\left(v_{1}^{\prime \prime}\right)=.15$, $\sigma_{2}\left(v_{2}^{\prime \prime}\right)=.5$. The corresponding incidence matrices $M_{H_{1}}, M_{H_{2}}$ are given by

\begin{tabular}{|c||cc|}
\hline$M_{H_{1}}$ & $E_{1}^{\prime}$ & $E_{2}^{\prime}$ \\
\hline \hline$v_{1}^{\prime}$ & $(.25, .2)$ & $(.3, .41)$ \\
$v_{2}^{\prime}$ & $(.5, .4)$ & $(.39, .6)$ \\
\hline
\end{tabular}

\begin{tabular}{|c||cc|}
\hline$M_{H_{2}}$ & $E_{1}^{\prime \prime}$ & $E_{2}^{\prime \prime}$ \\
\hline \hline$v_{1}^{\prime \prime}$ & $(.45, .3)$ & $(.41, .6)$ \\
$v_{2}^{\prime \prime}$ & $(.8, .5)$ & $(.0, .5)$ \\
\hline
\end{tabular}

Here $H_{1}$ is generalized strong intuitionistic fuzzy hypergraph and $H_{1}$ is generalized intuitionistic fuzzy hypergraph. Let $H=\left(V_{1} \times V_{2}, E_{1} \times E_{2}\right)$, $\rho=\rho_{1} \times \rho_{2}, \sigma=\sigma_{1} \times \sigma_{2}$ and $v_{i}^{\prime} v_{j}^{\prime \prime}=\left(v_{i}^{\prime}, v_{j}^{\prime \prime}\right)$ for all $i, j=1,2$. Then $\rho\left(v_{1}^{\prime} v_{1}^{\prime \prime}\right)=.3, \rho\left(v_{1}^{\prime} v_{2}^{\prime \prime}\right)=.3, \rho\left(v_{2}^{\prime} v_{1}^{\prime \prime}\right)=.45, \rho\left(v_{2}^{\prime} v_{2}^{\prime \prime}\right)=.5$ and $\sigma\left(v_{1}^{\prime} v_{1}^{\prime \prime}\right)=.2$, $\sigma\left(v_{1}^{\prime} v_{2}^{\prime \prime}\right)=.5, \sigma\left(v_{2}^{\prime} v_{1}^{\prime \prime}\right)=.4, \sigma\left(v_{2}^{\prime} v_{2}^{\prime \prime}\right)=.5$.

The corresponding incidence matrix $M_{H}$ is as follows:

\begin{tabular}{|c||c|c|c|c|}
\hline$M_{H}$ & $E_{1}^{\prime} \times E_{1}^{\prime \prime}$ & $E_{1}^{\prime} \times E_{2}^{\prime \prime}$ & $E_{2}^{\prime} \times E_{1}^{\prime \prime}$ & $E_{2}^{\prime} \times E_{2}^{\prime \prime}$ \\
\hline \hline$v_{1}^{\prime} v_{1}^{\prime \prime}$ & $(.25, .2)$ & $(.25, .2)$ & $(.3, .41)$ & $(.3, .6)$ \\
$v_{1}^{\prime} v_{2}^{\prime \prime}$ & $(.25, .5)$ & $(.0, .5)$ & $(.3, .5)$ & $(.0, .5)$ \\
$v_{2}^{\prime} v_{1}^{\prime \prime}$ & $(.45, .4)$ & $(.41, .6)$ & $(.39, .6)$ & $(.39, .6)$ \\
$v_{2}^{\prime} v_{2}^{\prime \prime}$ & $(.5, .5)$ & $(.0, .5)$ & $(.39, .6)$ & $(.0, .6)$ \\
\hline
\end{tabular}

Here it is easy to see that $H$ is a GSIFHG. 
Again, consider $H_{1}=\left(V_{1}, E_{1}\right)$ and $H_{2}=\left(V_{2}, E_{2}\right)$ where $V_{1}=\left\{v_{1}^{\prime}, v_{2}^{\prime}\right\}$, $E_{1}=\left\{E_{1}^{\prime}, E_{2}^{\prime}\right\}$ and $V_{2}=\left\{v_{1}^{\prime \prime}, v_{2}^{\prime \prime}\right\}, E_{2}=\left\{E_{1}^{\prime \prime}, E_{2}^{\prime \prime}\right\}$. Here $\rho_{1}, \sigma_{1}: V_{1} \rightarrow[0,1]$, $\rho_{2}, \sigma_{2}: V_{2} \rightarrow[0,1]$ are defined by $\rho_{1}\left(v_{1}^{\prime}\right)=.34, \rho_{1}\left(v_{2}^{\prime}\right)=.43, \sigma_{1}\left(v_{1}^{\prime}\right)=.02$, $\sigma_{1}\left(v_{2}^{\prime}\right)=.43$ and $\rho_{2}\left(v_{1}^{\prime \prime}\right)=.4, \rho_{2}\left(v_{2}^{\prime \prime}\right)=.33, \sigma_{2}\left(v_{1}^{\prime \prime}\right)=.1, \sigma_{2}\left(v_{2}^{\prime \prime}\right)=.39$.

The corresponding incidence matrices $M_{H_{1}}, M_{H_{2}}$ are given by

\begin{tabular}{|c||cc|}
\hline$M_{H_{1}}$ & $E_{1}^{\prime}$ & $E_{2}^{\prime}$ \\
\hline \hline$v_{1}^{\prime}$ & $(.34, .2)$ & $(.3, .02)$ \\
$v_{2}^{\prime}$ & $(.35, .43)$ & $(.43, .2)$ \\
\hline
\end{tabular}

\begin{tabular}{|c||cc|}
\hline$M_{H_{2}}$ & $E_{1}^{\prime \prime}$ & $E_{2}^{\prime \prime}$ \\
\hline \hline$v_{1}^{\prime \prime}$ & $(.35, .3)$ & $(.21, .6)$ \\
$v_{2}^{\prime \prime}$ & $(.33, .5)$ & $(.2, .5)$ \\
\hline
\end{tabular}

Here both $H_{1}$ and $H_{1}$ are GSIFHG. Let $H=\left(V_{1} \times V_{2}, E_{1} \times E_{2}\right), \rho=\rho_{1} \times \rho_{2}$, $\sigma=\sigma_{1} \times \sigma_{2}$ and $v_{i}^{\prime} v_{j}^{\prime \prime}=\left(v_{i}^{\prime}, v_{j}^{\prime \prime}\right)$ for all $i, j=1,2$. Then $\rho\left(v_{1}^{\prime} v_{1}^{\prime \prime}\right)=.34$, $\rho\left(v_{1}^{\prime} v_{2}^{\prime \prime}\right)=.33, \rho\left(v_{2}^{\prime} v_{1}^{\prime \prime}\right)=.4, \rho\left(v_{2}^{\prime} v_{2}^{\prime \prime}\right)=.33$ and $\sigma\left(v_{1}^{\prime} v_{1}^{\prime \prime}\right)=.1, \sigma\left(v_{1}^{\prime} v_{2}^{\prime \prime}\right)=.29$, $\sigma\left(v_{2}^{\prime} v_{1}^{\prime \prime}\right)=.43, \sigma\left(v_{2}^{\prime} v_{2}^{\prime \prime}\right)=.39$.

The corresponding incidence matrix $M_{H}$ is as follows:

\begin{tabular}{|c||c|c|c|c|}
\hline$M_{H}$ & $E_{1}^{\prime} \times E_{1}^{\prime \prime}$ & $E_{1}^{\prime} \times E_{2}^{\prime \prime}$ & $E_{2}^{\prime} \times E_{1}^{\prime \prime}$ & $E_{2}^{\prime} \times E_{2}^{\prime \prime}$ \\
\hline \hline$v_{1}^{\prime} v_{1}^{\prime \prime}$ & $(.2, .25)$ & $(.25, .2)$ & $(.23, .4)$ & $(.3, .2)$ \\
$v_{1}^{\prime} v_{2}^{\prime \prime}$ & $(.25, .3)$ & $(1, .5)$ & $(.3, .6)$ & $(.32, .5)$ \\
$v_{2}^{\prime} v_{1}^{\prime \prime}$ & $(.3, .46)$ & $(.14, .6)$ & $(.39, .5)$ & $(.35, .58)$ \\
$v_{2}^{\prime} v_{2}^{\prime \prime}$ & $(.3, .45)$ & $(.02, .4)$ & $(.17, .66)$ & $(.29, .6)$ \\
\hline
\end{tabular}

Here it is easy to see that $H$ is a GIFHG but not a GSIFHG.

Proposition 3.12. If $H_{1}$ and $H_{2}$ are generalized intuitionistic fuzzy hypergraphs, then $H_{1} \times H_{2}$ is a generalized intuitionistic fuzzy hypergraphs.

Proof. Let $H_{1}=\left(V_{1}, E_{1}\right)$ and $H_{2}=\left(V_{2}, E_{2}\right)$ be two GIFHGs, where

$$
\begin{array}{ll}
V_{1}=\left\{x_{1}, x_{2}, \ldots, x_{n}\right\}, & \rho_{1}, \sigma_{1}: V_{1} \rightarrow[0,1], \\
V_{2}=\left\{y_{1}, y_{2}, \ldots, y_{m}\right\}, & \rho_{2}, \sigma_{2}: V_{2} \rightarrow[0,1]
\end{array}
$$

and

$$
\begin{array}{ll}
E_{1}=\left\{\left(\mu_{11}, \nu_{11}\right),\left(\mu_{12}, \nu_{12}\right), \ldots,\left(\mu_{1 k}, \nu_{1 k}\right)\right\}, & \mu_{1 i}, \nu_{1 i}: V_{1} \rightarrow[0,1] \\
E_{2}=\left\{\left(\mu_{21}, \nu_{21}\right),\left(\mu_{22}, \nu_{22}\right), \ldots,\left(\mu_{2 p}, \nu_{2 p}\right)\right\}, & \mu_{2 j}, \nu_{2 j}: V_{2} \rightarrow[0,1]
\end{array}
$$

for all $i=1,2, \ldots, k$ and $j=1,2, \ldots, p$.

Then $H_{1} \times H_{2}=\left(V_{1} \times V_{2}, E_{1}, E_{2}\right)$, where $E_{1} \times E_{2}=\left\{\left(\left(\mu_{11} \times \mu_{21}\right),\left(\nu_{11} \times\right.\right.\right.$ $\left.\left.\left.\nu_{21}\right)\right), \ldots,\left(\left(\mu_{11} \times \mu_{2 p}\right),\left(\nu_{11} \times \nu_{2 p}\right)\right), \ldots,\left(\left(\mu_{1 k} \times \mu_{2 p}\right),\left(\nu_{1 k} \times \nu_{2 p}\right)\right)\right\}, \mu_{1 i} \times \mu_{2 j}$ and $\nu_{1 i} \times \nu_{2 j}$ are defined by the condition (ii) of the Definition 3.9. We see that

$$
\begin{array}{ll}
\bigvee_{r=1}^{k} \mu_{1 r}\left(x_{i}\right) \leq \rho_{1}\left(x_{i}\right), & \bigwedge_{r=1}^{k} \nu_{1 r}\left(x_{i}\right) \geq \sigma_{1}\left(x_{i}\right), \quad \forall i=1,2, \ldots, n \\
\bigvee_{s=1}^{p} \mu_{2 s}\left(y_{j}\right) \leq \rho_{2}\left(y_{j}\right), & \stackrel{\bigwedge_{s=1}^{p}}{\wedge} \nu_{2 s}\left(y_{j}\right) \geq \sigma_{2}\left(y_{j}\right), \quad j=1,2, \ldots, m .
\end{array}
$$


By the condition $(i i)$ of the Definition 3.9, we have

$$
\begin{aligned}
\left(\mu_{1 r} \times \mu_{2 s}\right)\left(x_{i}, y_{j}\right) & =\mu_{1 r}\left(x_{i}\right) \wedge \mu_{2 s}\left(y_{j}\right), \quad \forall i, j, \\
\left(\nu_{1 r} \times \nu_{2 s}\right)\left(x_{i}, y_{j}\right) & =\nu_{1 r}\left(x_{i}\right) \vee \nu_{2 s}\left(y_{j}\right), \quad \forall i, j .
\end{aligned}
$$

This implies that

$$
\begin{aligned}
\bigvee_{s=1}^{p} \bigvee_{r=1}^{k}\left(\mu_{1 r} \times \mu_{2 s}\right)\left(x_{i}, y_{j}\right) & =\bigvee_{s=1}^{p} \bigvee_{r=1}^{k}\left(\mu_{1 r}\left(x_{i}\right) \wedge\left(\mu_{2 s}\left(y_{j}\right)\right)\right. \\
=\left(\bigvee _ { r = 1 } ^ { k } ( \mu _ { 1 r } ( x _ { i } ) ) \wedge \left(\bigvee_{s=1}^{p}\left(\mu_{2 s}\left(y_{j}\right)\right)\right.\right. & \leq \rho_{1}\left(x_{i}\right) \wedge \rho_{2}\left(y_{j}\right) \\
& =\left(\rho_{1} \times \rho_{2}\right)\left(x_{i}, y_{j}\right), \quad \forall i, j,
\end{aligned}
$$

i.e.,

$$
\bigvee_{s=1}^{p} \bigvee_{r=1}^{k}\left(\mu_{1 r} \times \mu_{2 s}\right)\left(x_{i}, y_{j}\right) \leq\left(\rho_{1} \times \rho_{2}\right)\left(x_{i}, y_{j}\right), \quad \forall i, j
$$

Similarly we have,

$$
\stackrel{p}{\wedge} \stackrel{k}{\wedge}\left(\nu_{1 r} \times \nu_{2 s}\right)\left(x_{i}, y_{j}\right) \geq\left(\sigma_{1} \times \sigma_{2}\right)\left(x_{i}, y_{j}\right), \quad \forall i, j .
$$

This completes the proof.

Proposition 3.13. If $H_{1}$ and $H_{2}$ are the strong intuitionistic fuzzy hypergraphs, then $H_{1} \times H_{2}$ is a strong intuitionistic fuzzy hypergraph.

Proof. Proof is same as that of the Proposition 3.12.

Proposition 3.14. If $H_{1} \times H_{2}$ is GSIFHG then at least $H_{1}$ or $H_{2}$ must be strong.

Proof. Let $H_{1}=\left(V_{1}, E_{1}\right)$ and $H_{2}=\left(V_{2}, E_{2}\right)$ be two GIFHGs, where $V_{1}=$ $\left\{x_{1}, x_{2}, \ldots, x_{n}\right\}, V_{2}=\left\{y_{1}, y_{2}, \ldots, y_{m}\right\}, \rho_{1}, \sigma_{1}: V_{1} \rightarrow[0,1], \rho_{2}, \sigma_{2}: V_{2} \rightarrow$ $[0,1]$ and $E_{1}=\left\{\left(\mu_{11}, \nu_{11}\right),\left(\mu_{12}, \nu_{12}\right), \ldots,\left(\mu_{1 k}, \nu_{1 k}\right)\right\}, E_{2}=\left\{\left(\mu_{21}, \nu_{21}\right)\right.$, $\left.\left(\mu_{22}, \nu_{22}\right), \ldots,\left(\mu_{2 p}, \nu_{2 p}\right)\right\}, \mu_{1 i}, \nu_{1 i}: V_{1} \rightarrow[0,1] ; \mu_{2 j}, \nu_{2 j}: V_{2} \rightarrow[0,1]$ for all $i=1,2, \ldots, k$ and $j=1,2, \ldots, p$.

Then $H_{1} \times H_{2}=\left(V_{1} \times V_{2}, E_{1}, E_{2}\right)$, where $E_{1} \times E_{2}=\left\{\left(\left(\mu_{11} \times \mu_{21}\right),\left(\nu_{11} \times\right.\right.\right.$ $\left.\left.\left.\nu_{21}\right)\right), \ldots,\left(\left(\mu_{11} \times \mu_{2 p}\right),\left(\nu_{11} \times \nu_{2 p}\right)\right), \ldots,\left(\left(\mu_{1 k} \times \mu_{2 p}\right),\left(\nu_{1 k} \times \nu_{2 p}\right)\right)\right\}, \mu_{1 i} \times \mu_{2 j}$ and $\nu_{1 i} \times \nu_{2 j}$ are defined by the condition $(i i)$ of the Definition 3.9.

We suppose that $H_{1} \times H_{2}$ is GSIFHG but both $H_{1}$ and $H_{2}$ are not strong. Then from the condition (iv) of the Definition 3.1, we have

$$
\begin{array}{ll}
\bigvee_{r=1}^{k} \mu_{1 r}\left(x_{i}\right)<\rho_{1}\left(x_{i}\right), & \bigvee_{s=1}^{p} \mu_{2 s}\left(y_{j}\right)<\rho_{2}\left(y_{j}\right), \\
\bigwedge_{r=1}^{k} \nu_{1 r}\left(x_{i}\right)>\sigma_{1}\left(x_{i}\right), & {\stackrel{\wedge}{p=1} \nu_{2 s}\left(y_{j}\right)>\sigma_{2}\left(y_{j}\right),}^{p},
\end{array}
$$

forall $i=1,2, \ldots, n$ and $j=1,2, \ldots, m$. 
Again, by by the condition (ii) of the Definition 3.9, we get,

$$
\begin{aligned}
\left(\mu_{1 r} \times \mu_{2 s}\right)\left(x_{i}, y_{j}\right) & =\mu_{1 r}\left(x_{i}\right) \wedge \mu_{2 s}\left(y_{j}\right), \quad \forall i, j, \\
\left(\nu_{1 r} \times \nu_{2 s}\right)\left(x_{i}, y_{j}\right) & =\nu_{1 r}\left(x_{i}\right) \vee \nu_{2 s}\left(y_{j}\right), \quad \forall i, j .
\end{aligned}
$$

This implies that

$$
\begin{aligned}
\bigvee_{s=1}^{p} \bigvee_{r=1}^{k}\left(\mu_{1 r} \times \mu_{2 s}\right)\left(x_{i}, y_{j}\right) & =\bigvee_{s=1}^{p} \bigvee_{r=1}^{k}\left(\mu_{1 r}\left(x_{i}\right) \wedge\left(\mu_{2 s}\left(y_{j}\right)\right)\right. \\
=\left(\bigvee _ { r = 1 } ^ { k } ( \mu _ { 1 r } ( x _ { i } ) ) \wedge \left(\bigvee_{s=1}^{p}\left(\mu_{2 s}\left(y_{j}\right)\right)\right.\right. & <\rho_{1}\left(x_{i}\right) \wedge \rho_{2}\left(y_{j}\right) \\
& =\left(\rho_{1} \times \rho_{2}\right)\left(x_{i}, y_{j}\right), \quad \forall i, j
\end{aligned}
$$

i.e.,

$$
\bigvee_{s=1}^{p} \bigvee_{r=1}^{k}\left(\mu_{1 r} \times \mu_{2 s}\right)\left(x_{i}, y_{j}\right)<\left(\rho_{1} \times \rho_{2}\right)\left(x_{i}, y_{j}\right), \quad \forall i, j
$$

Similarly we have,

$$
\stackrel{p}{\wedge} \stackrel{k}{s=1}_{r=1}^{\wedge}\left(\nu_{1 r} \times \nu_{2 s}\right)\left(x_{i}, y_{j}\right)>\left(\sigma_{1} \times \sigma_{2}\right)\left(x_{i}, y_{j}\right), \quad \forall i, j .
$$

That is, $H_{1} \times H_{2}$ is not a GSIFHG, a contradiction. Hence at least one of $H_{1}, H_{2}$ must be strong.

Definition 3.15. Let $H_{1}=\left(V_{1}, E_{1}\right)$ and $H_{2}=\left(V_{2}, E_{2}\right)$ be two GIFHGs, where $V_{1}=\left\{x_{1}, x_{2}, \ldots, x_{n}\right\}, V_{2}=\left\{y_{1}, y_{2}, \ldots, y_{m}\right\}, \rho_{1}, \sigma_{1}: V_{1} \rightarrow[0,1]$, $\rho_{2}, \sigma_{2}: V_{2} \rightarrow[0,1]$ and $E_{1}=\left\{\left(\mu_{11}, \nu_{11}\right),\left(\mu_{12}, \nu_{12}\right), \ldots,\left(\mu_{1 k}, \nu_{1 k}\right)\right\}, E_{2}=$ $\left\{\left(\mu_{21}, \nu_{21}\right),\left(\mu_{22}, \nu_{22}\right), \ldots,\left(\mu_{2 p}, \nu_{2 p}\right)\right\}, \mu_{1 i}, \nu_{1 i}: V_{1} \rightarrow[0,1] ; \mu_{2 j}, \nu_{2 j}: V_{2} \rightarrow$ $[0,1]$ for all $i=1,2, \ldots, k$ and $j=1,2, \ldots, p$.

The union $H_{1} \cup H_{2}=\left(V_{1} \cup V_{2}, E_{1} \cup E_{2}\right)$ of $H_{1}$ and $H_{2}$ is defined as follows:

$$
\begin{aligned}
& \left(\rho_{1} \cup \rho_{2}\right)(x)=\rho_{1}(x) \text { if } x \in V_{1} \text { and } x \notin V_{2}, \\
& \left(\rho_{1} \cup \rho_{2}\right)(x)=\rho_{2}(x) \text { if } x \in V_{2} \text { and } x \notin V_{1}, \\
& \left(\rho_{1} \cup \rho_{2}\right)(x)=\max \left(\rho_{1}(x), \rho_{2}(x)\right) \text { if } x \in V_{1} \cap V_{2} ; \\
& \left(\sigma_{1} \cup \sigma_{2}\right)(x)=\sigma_{1}(x) \text { if } x \in V_{1} \text { and } x \notin V_{2}, \\
& \left(\sigma_{1} \cup \sigma_{2}\right)(x)=\sigma_{2}(x) \text { if } x \in V_{2} \text { and } x \notin V_{1}, \\
& \left(\sigma_{1} \cup \sigma_{2}\right)(x)=\min \left(\sigma_{1}(x), \sigma_{2}(x)\right) \text { if } x \in V_{1} \cap V_{2} ;
\end{aligned}
$$




$$
\begin{aligned}
& \left(\mu_{1 i} \cup \mu_{2 j}\right)(x)=\mu_{1 i}(x) \text { if } x \in V_{1} \text { and } x \notin V_{2}, \\
& \left(\mu_{1 i} \cup \mu_{2 j}\right)(x)=\mu_{2 j}(x) \text { if } x \in V_{2} \text { and } x \notin V_{1}, \\
& \left(\mu_{1 i} \cup \mu_{2 j}\right)(x)=\max \left(\mu_{1 i}(x), \mu_{2 j}(x)\right) \text { if } x \in V_{1} \cap V_{2} ; \\
& \left(\nu_{1 i} \cup \nu_{2 j}\right)(x)=\nu_{1 i}(x) \text { if } x \in V_{1} \text { and } x \notin V_{2}, \\
& \left(\nu_{1 i} \cup \nu_{2 j}\right)(x)=\nu_{2 j}(x) \text { if } x \in V_{2} \text { and } x \notin V_{1}, \\
& \left(\nu_{1 i} \cup \nu_{2 j}\right)(x)=\min \left(\nu_{1 i}(x), \nu_{2 j}(x)\right) \text { if } x \in V_{1} \cap V_{2} .
\end{aligned}
$$

Example 3.16. Consider the generalized intuitionistic fuzzy hypergraphs $G=\left(V_{G}, E_{G}\right)$ and $S=\left(V_{S}, E_{S}\right)$ where $V_{G}=\left\{x_{1}, x_{2}\right\}, E_{G}=\left\{E_{1}, E_{2}\right\}$ and $V_{S}=\left\{v_{1}, x_{2}\right\}, E_{S}=\left\{E_{1}^{\prime}, E_{2}^{\prime}\right\}$. Here $\rho_{1}, \sigma_{1}: V_{G} \rightarrow[0,1], \rho_{2}, \sigma_{2}: V_{S} \rightarrow[0,1]$ given by $\rho_{1}\left(x_{1}\right)=.5, \rho_{1}\left(x_{2}\right)=.1, \sigma_{1}\left(x_{1}\right)=.2, \sigma_{1}\left(x_{2}\right)=.23$ and $\rho_{2}\left(v_{1}\right)=$ $.39, \rho_{2}\left(x_{2}\right)=.32, \sigma_{2}\left(v_{1}\right)=.02, \sigma_{2}\left(x_{2}\right)=.29$.

The corresponding incidence matrices $M_{G}$ and $M_{S}$ are given by

\begin{tabular}{|c||cc|}
\hline$M_{G}$ & $E_{1}$ & $E_{2}$ \\
\hline \hline$x_{1}$ & $(.5, .3)$ & $(.2, .2)$ \\
$x_{2}$ & $(.02, .22)$ & $(.1, .23)$ \\
\hline
\end{tabular}

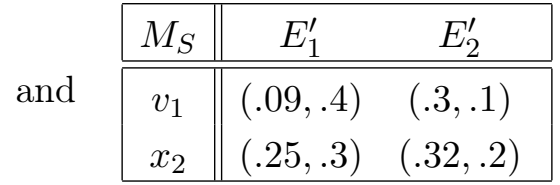

Here we see that both $G$ and $S$ are generalized intuitionistic fuzzy hypergraphs. Let $\rho=\rho_{1} \cup \rho_{2}, \sigma=\sigma_{1} \cup \sigma_{2}, E_{1}^{\prime \prime}=E_{1} \cup E_{1}^{\prime}, E_{2}^{\prime \prime}=E_{1} \cup E_{2}^{\prime}$, $E_{3}^{\prime \prime}=E_{2} \cup E_{1}^{\prime}, E_{4}^{\prime \prime}=E_{2} \cup E_{2}^{\prime}$.

Then we have $\rho\left(x_{1}\right)=.5, \rho\left(x_{2}\right)=.32, \rho\left(v_{1}\right)=.39, \sigma\left(x_{1}\right)=.2, \sigma\left(x_{2}\right)=$ $.23, \sigma\left(v_{1}\right)=.02$.

The corresponding incidence matrix $M_{G \cup S}$ is given by

\begin{tabular}{|c||cccc|}
\hline$M_{G \cup S}$ & $E_{1}^{\prime \prime}$ & $E_{2}^{\prime \prime}$ & $E_{3}^{\prime \prime}$ & $E_{4}^{\prime \prime}$ \\
\hline \hline$x_{1}$ & $(.5, .3)$ & $(.5, .3)$ & $(.2, .2)$ & $(.2, .2)$ \\
$x_{2}$ & $(.25, .22)$ & $(.32, .2)$ & $(.25, .23)$ & $(.32, .2)$ \\
$v_{1}$ & $(.09, .4)$ & $(.3, .1)$ & $(.09, .4)$ & $(.3, .1)$ \\
\hline
\end{tabular}

It is easy to verify that $M_{G \cup S}$ is a generalized intuitionistic fuzzy hypergraph but not strong.

Proposition 3.17. If $H_{1}$ and $H_{2}$ are generalized intuitionistic fuzzy hypergraphs, then $\mathrm{H}_{1} \cup \mathrm{H}_{2}$ is a generalized intuitionistic fuzzy hypergraph.

Conclusion 3.18. In this paper, the concept of intuitionistic fuzzy hyper graph has been generalized by considering intuitionistic fuzzy vertex instead of crisp vertex set and also considering interrelation between intuitionistic fuzzy vertex and family of intuitionistic fuzzy edges. Further one can use this concept to analyze the structure of a system and to represent a partition, covering and clustering. 


\section{REFERENCES}

[1] A. Kaufmann, Introduction a la Theory des Sous - Ensemble Flous, Vol. 1, Masson: Paris, 1977.

[2] A. Rosenfeld, Fuzzy graphs, Fuzzy sets and their Applications ,(L.A. Zadeh, K.S. Fu, M. Shimura, Eds) Academic Press, New York, 1975, 77-95.

[3] C. Berge, Graphes et Hypergraphes, Dunod:Paris, 1970.

[4] K. Atanassov, Intuitionistic fuzzy sets, Fuzzy Sets and Systems, 20 (1986), 87-96.

[5] K. Atanassov, Intuitionistic fuzzy sets, Theory, Applications, Studies in fuzziness and computing, Heidelberg, New York, physica-Verl., 1999.

[6] L.A. Zadeh, Fuzzy sets, Information Control 8 (1965), 338-353.

[7] M.Akram, B.Davvaz, Strong Intuitionistic fuzzy graphs, Filomat 26:1 (2012), 177196.

[8] R. Parvathi, S. Thilagavathi, M.G. Karunambigai, Intuitionistic Fuzzy Hypergraphs, Cybernetics And Information Technologies 9(2) (2009), 46-53.

[9] S.K. De, R. Biswas and A.R. Roy An application of intuitionistic Fuzzy set in medical diagnosis , Fuzzy Sets and Systems, 117 (2001), 209-213.

[10] H. Lee-Kwang, K.-M. Lee, An application of intuitionistic Fuzzy set in medical diagnosis, Fuzzy Hypergraph and Fuzzy Partition, IEEE Transactions on Systems,Man, and Cybernetics, 25(1) (1995), 196-201.

\section{T.K. Samanta}

Department of Mathematics

Uluberia College

Uluberia, Howrah - 711315

West Bengal, India

E-mail address: mumpu_tapas5@yahoo.co.in

\section{Sumit Mohinta}

Department of Mathematics

Sudhir Memorial Institute

KOLKATA - 700132

West Bengal, India

E-mail address: sumit.mohinta@yahoo.com 